\title{
Detection of Chlamydia Trachomatis infection among symptomatic women of reproductive age group attending the gynaecology OPD in a tertiary care centre and risk factors associated with the infection
}

\author{
Meena Dias ${ }^{1, *}$, Rakhshitha Puroshotam Eashwernath ${ }^{2}$ \\ ${ }^{1}$ Associate Professor, ${ }^{2}$ Resident, Dept. of Microbiology, Father Muller Medical College, Mangalore, Karnataka, India
}

*Corresponding Author:

Email: drmeenadias@gmail.com

\begin{abstract}
Chlamydia trachomatis infection is the most common sexually transmitted bacterial infection worldwide, affecting women more than men. Majority being asymptomatic, this infection goes unnoticed and will have a major impact on the reproductive health of women resulting in morbidity. It also increases the risk of HIV.

Objective: To detect the Chlamydial infection in symptomatic women of reproductive age group and to identify the possible risk factors.

Materials and Methods: An endocervical swabs were collected from 100 symptomatic patients of reproductive age group and subjected to direct fluorescent antibody test (DFA).

Results: The C.trachomatis infection was $20 \%$, with a higher positivity in the age group of 26- 30 years. The most presenting complaint was vaginal discharge. A significant past history of PID, coppet T insertion, IUD/still birth was noted. A $20 \%$ coinfection with candiadiasis was also observed.

Conclusion: As the occurence of Chlamydia trachomatis was substantially high in our study, effective strategies to implement screening programmes should be initiated as a routine diagnostic test for all women of reproductive age group, thereby preventing severe sequelae by providing timely management.
\end{abstract}

Keywords: Chlamydia trachomatis, Percentage, occurrence, Direct fluorescent antibody test, Risk factors.

\section{Introduction}

Chlamydia trachomatis is the most common sexually transmitted bacterial infection worldwide and women carry the major burden of the disease. ${ }^{1}$ It is also a leading preventable cause of Pelvic Inflammatory Disease (PID) and female infertility globally. ${ }^{2}$ Majority of the patients are asymptomatic, thereby providing an ongoing reservoir of infection capable of transmitting it to their sexual partners.

These undiagnosed infections often present as PID leading to severe complications like ectopic pregnancy, tubal factor infertility and enhances the risk of transmission of Human immunodeficiency virus (HIV) and other sexually transmitted infections (STIs). ${ }^{3}$ It is also a risk factor for cervical carcinoma. ${ }^{4}$ Although Chlamydia trachomatis is an established cause of infertility, there is an increasing prevalence of this infection in developing countries which is attributed to the fact that there is a lack of effective screening programmes, limited resources and cost-effective diagnostic tests. These chlamydial infections are a major health care concern in women more than men since the manifestations and consequences are more detrimental to the reproductive health of women. ${ }^{5}$ Worldwide, the magnitude of morbidity associated with sexually transmitted chlamydial infections is enormous.

Chlamydial infections can be easily treated with oral antibiotics, but the percentage of people being symptomatic are very few thereby leading to long term sequelae, especially in women if left untreated. Therefore this study was carried out to evaluate the occurrence of Chlamydia trachomatis infection in symptomatic women by using a cost effective and a simple diagnostic procedure like the direct fluorescent assay (DFA).

\section{Materials and Methods}

The prospective study was carried out in the Department of Microbiology of a tertiary care teaching hospital, for a period of one and a half years from October 2014 to April 2016 after obtaining approval from the Institutional Ethics Committee. Written informed consent was obtained from all the participants willing to participate in the study. A total of 100 endocervical swabs were collected from all women of reproductive age group (18-40 years) attending the Gynaecology outpatient department (OPD) and inpatients (IP) and sent to the microbiology laboratory for further processing.

Demographic data like age, socioeconomic status, occupation, marital history, presenting complaints, obstetric history, and menstrual history were collected. Relevant clinical details and investigations were also obtained. Only those women of reproductive age group (18-40 years) who are sexually active, presence of active vaginal or cervical discharge, women complaining of infertility, women complaining of pain abdomen with significant menstrual history ( menorrhagia/oligomennorhea/irregular menses) were included in the study. All pregnant women, women below < 18 years and above > 40 years and menstruating women were excluded from the study. 
A speculum was passed and an endocervical specimen was collected using sterile cotton tipped swab and was placed in a screw capped polypropylene tube. It was then sent to the microbiology laboratory immediately for further evaluation by Direct Fluorescent Antibody Test (DFA).

The cotton swab received at the laboratory was immediately processed. It was rolled onto a sterile glass slide evenly and firmly and left to air dry. The slide was then flooded with $0.5 \mathrm{ml}$ methanol fixative and was allowed to evaporate completely. The process was speeded up by tipping the slide after 5 minutes to drain the excess fixative. The slides were then stored at $-20^{0}$ $\mathrm{C}$ until staining.

MicroTrak Chlamydia trachomatis (Trinity Biotech) direct specimen test kit was used for staining the slides and procedure was followed according to manufacturer instructions. and MicroTrak Chlamydia trachomatis direct specimen test control slide pack with positive and negative control slides were also stained each time the specimen slides were stained. Before staining, the reagents, control and specimen slides were brought down to room temperature.The reagent is a fluorescein-isothiocyanate dye conjugated with monoclonal antibodies which is highly specific for the MOMP of Chlamydia trachomatis.

The slides were then placed on a staining rack and $30 \mu 1$ of reagent was added to the control and fixed specimen slides and incubated for 15 minutes at room temperature in a humidified chamber. It was then washed with distilled water. Excess water was removed by blotting around the smear with a tissue paper and then was air dried. A drop of mounting fluid was added to the center of the slide and a cover slip was placed on the top of the drop.

Stained slides were then viewed under a fluorescent microscope. 40x was used for screening and $100 x$ was used for confirming the morphology. Specimens were examined for apple green coloured elementary bodies contrasted against the reddish brown background of the counterstained cells. Positive control slides were used as a guide to the size, shape and staining pattern of elementary bodies.

Presence of 10 or more chlamydial elementary bodies was considered positive. When no such structures were seen, the sample was considered negative.

\section{Statistical Analysis}

Collected data was analyzed by frequency, percentage, Chi Square test and Fischer Exact test. SPSS version 20.0 was used for all the statistical procedures.

\section{Results}

Out of 100 samples processed, 20 were positive for Chlamydia trachomatis infection. 10 or more EB's per smear were seen in all the 20 cases. No doubtful positive cases were observed in our study. The highest positivity was seen in the age group 26-30 years $9(45 \%)$ followed by $>31$ years $6(30 \%)$ and $20-25$ years $5(25 \%)$. Chlamydia was detected more commonly in women with a parity index of more than one 14(70\%) as compared to nulliparous women $6(30 \%)$.

The most common presenting complaint was vaginal discharge. Some presented with significant menstrual disturbances like irregular menses/ menorrhagia/ oligomenorrhea. Table 1 shows presenting complaints of patients. In our study population of 100 women, 2 patients had a history of infertility, out of which only one was detected with Chlamydia.

Out of the 20 patients who were positive for Chlamydia, 14 patients had a significant past history of PID- single or multiple episodes, copper T insertion, bad obstetric history. Bad obstetric history included abortions seen in 3(15\%), intrauterine death in $2(10 \%)$ and still birth seen in $1(5 \%)$ patient There was no significant history in 6 patients (Table 2). This had a significant $\mathrm{p}$ value of 0.036 .

The coinfection with Chlamydia trachomatis with Candidiasis was seen in 4(20\%) cases. Only one patient was positive for HIV.

All the 20 patients that were detected for Chlamydia were sexually active. Onset of sexual activity in our study was observed to be higher in the age group of 26-30 years. May be this is the reason for highest positivity in this age group. None gave history of multiple sexual partners.

Table 1: Presenting complaints associated with positive genital Chlamydia infection

\begin{tabular}{|l|c|c|}
\hline \multicolumn{1}{|c|}{ Presenting complaints } & Chlamydia positive & \multirow{2}{*}{$\mathrm{P}=0.724$} \\
\hline Vaginal discharge & $11(55 \%)$ & \\
\cline { 1 - 1 } $\begin{array}{l}\text { Pain abdomen with significant } \\
\text { disturbance in menstrual history }\end{array}$ & $8(40 \%)$ & \\
\hline Primary infertility & $1(5 \%)$ & \\
\hline
\end{tabular}


Table 2: Significant risk factors among women detected positive for C.trachomatis

\begin{tabular}{|c|c|c|}
\hline Relevant past history & $\begin{array}{c}\text { Chlamydia } \\
\text { detected }\end{array}$ & \multirow{2}{*}{$\mathrm{P}=0.036$} \\
\hline PID & $4(20 \%)$ & \\
\hline Copper T insertion & $4(20 \%)$ & \\
\hline Bad obstetric history & $6(30 \%)$ & \\
\hline
\end{tabular}

\section{Discussion}

Chlamydia trachomatis is a Gram-negative obligate intracellular bacterium with an incubation period of 7 to 21 days and a growth cycle of approximately 48 hours. $^{5}$ C. trachomatis has a worldwide distribution, affecting both sexes, but has a much greater impact on females than on males. It particularly affects young women and sexually active adolescents. Unfortunately, chlamydial infections may, in many cases, remain silent. Timely detection and effective management of cervical infection due to Chlamydia trachomatis in women provides critical interventional opportunities.

The gold standard for diagnosis of Chlamydia trachomatis is by culture in cell lines. But as it is highly expensive in addition to its low sensitivity and laborious procedure, a search for better and alternative diagnostic strategies was initiated so that it can be more feasible in a resource restricted setting. Among these was the antigen detection by DFA which has a sensitivity of $90 \%$ and $98-99 \%$ specificity. ${ }^{6}$ This high specificity is due to its dependence on visualization of the brightly stained elementary bodies which implies the presence of an active chlamydial infection. Also the procedure is rapid, making it an ideal test in laboratory set up.

The percentage of Chlamydia trachomatis infection in women of reproductive age group attending the Gynaecology department in this study using the DFA technique was found to be $20 \%$. There are various studies which have reported the prevalence in a population. A study conducted in New Delhi revealed a $8.8 \%$ prevalence, ${ }^{7}$ in contrast to another study conducted in Malhotra $M$ et al in which $19.9 \%$ prevalence was observed. ${ }^{8}$ A higher prevalence of 24.5\% was reported in a study conducted in Chennai, India using DFA technique by Joyee AG et al. ${ }^{9}$ A study conducted in Nigeria by Ikeme AC et al, also showed a high prevalence of $29.4 \% .{ }^{10}$ Study conducted by Sood et al showed a positivity of $11.34 \%$ by DFA technique. ${ }^{11}$ Another study by Sachadeva et al reported a figure of $22.9 \%$ positivity in symptomatic female patients attending the Gynaecology department of a tertiary care center. ${ }^{12}$

We observed detection rate was higher in the age group 26-30 years showing a $45 \%$ rate, $30 \%$ in the age group $>31$ years and $25 \%$ among women of 20-25 years. Age is the most important risk factor for chlamydial infection. The burden of Chlamydia appears higher among women especially those of younger age.
Among women, highest age- specific rates of reported Chlamydia in 2008 were $3,275.8$ cases per 100,000 females $(3.3 \%)$ among 15 to 19 years of age and $3,179.9$ cases per 100,000 females (3.2\%) among 20 to 24 years of age. ${ }^{1} \mathrm{CDC}$ recommends routine screening of all sexually active women of $<25$ years of age ${ }^{6}$ as there is a high risk in young women due to behavioral characteristics and also increased exposure of cervical columnar epithelium may enhance the ability of the organism to infect new cells. In UK, it has been reported that $10.3 \%$ of women $<25$ years of age are infected with Chlamydia. ${ }^{7}$ In a study conducted in Nicaragua by Hermann B et al, the mean age was found to be 27.3 years and majority $(30 \%)$ were married..$^{13}$ In another study the highest rate of chlamydial infection was found to be in the 20-30 years age group. ${ }^{8}$ One study conducted in Chennai, India, by Prathiba et al, ${ }^{14}$ showed similar results, wherein the highest percentage of Chlamydia positive patients was seen in the age group 26-30 years (34.6\%), which is almost similar to the data obtained in our study. Also all the women who were detected positive for Chlamydia in this study were married. In a study of 1800 women by Zenilman JM, the prevalence of chlamydial infection among married was $1 \% .{ }^{15}$ In another study conducted by Sood et al, the mean age was 29.7 years, with $73 \%$ in the age group of 15-34 years. ${ }^{11}$

The age of the patients in this study could not be associated with most of the studies because the highest positivity was seen in the age group 26-30 years. Probable explanation could be because of late onset of sexual activity observed in our population. CDC has showed that there is a high prevalence of chlamydial detection rate of $4.7 \%$ among sexually active females aged 14-24 years. ${ }^{[6]}$ There is a strong correlation found with age at onset of sexual activity with C.trachomatis infection. ${ }^{[14]}$ An inverse trend related age and prevalence of infection was found in this study, but statistically no significance was associated.

The most common presenting complaint by women in our study was vaginal discharge, seen in 55\% of the patients. A study by Sood et al, India, showed vaginal discharge to be the most common presenting complaint, seen in $91.75 \%$ patients. ${ }^{11}$ Nessa et al ${ }^{16}$ showed major cases $49.6 \%$ had excessive vaginal discharge. Hermann $B$ et $\mathrm{al}^{13}$ in Nicaragua, showed $64 \%$ women complaining with vaginal discharge. Mohan DG et al, ${ }^{17}$ showed $33.3 \%$ women complaining of vaginal discharge who were detected positive for Chlamydia. The strongest association was found with vaginal 
discharge, thus supporting the use of this symptom in the syndromic algorithm for the management of vaginal discharge that is recommended by WHO.

The next complaint was lower abdominal pain associated with significant disturbance in menstrual history (menorrhagia/irregular bleeding/oligomenorrhea) seen in $40 \%$ of the patients. Nessa et al showed $48.4 \%$ of the patients complaining with lower abdominal pain, which is closer to our study. ${ }^{16} \mathrm{~A}$ study conducted in North India by Dhawan et al, showed $10.5 \%$ came with a complaint of abdominal pain. ${ }^{18}$ Study done in Nicaragua by Hermann B et al, showed a rate of $55 \% .^{13}$

Chlamydia was detected only in 1 patient (5\%) presenting with primary infertility. She had a history of multiple episodes of PID which could be attributed to the development of infertility. A study by Dhawan et al, conducted only in infertile female patients, showed a chlamydial detection rate of $9 \% .{ }^{18}$

Out of the 20 patients that were detected positive for Chlamydia, 14 had a significant past history. Majority had a history of PID (20\%); with one or multiple episodes in the past, showing that probably untreated chlamydial infections in the past lead to the development of PID which if left untreated might go into further complications leading to infertility. ${ }^{19} 20 \%$ had a history of copper $\mathrm{T}$ insertion, which again is a major risk factor for chlamydial infection. Insertion of intra uterine devices when not done under aseptic precautions might also lead to PID. Another study by Herman B, et al, showed a prevalence rate of $1.8 \%$ in women with intra uterine devices. ${ }^{13} 30 \%$ had bad obstetric history wherein which, $15 \%$ had a history of unexplained abortions in the first trimester. Study by Visnovsky $\mathrm{J}$ et al, ${ }^{20}$ showed a significant correlation between chlamydial positivity and first trimester abortions of about $15.2 \%$ which is similar to what we obtained in our study. Dhawan et al ${ }^{18}$ showed a history of abortions in $39 \%$ of the women who were detected positive for Chlamydia.

$10 \%$ of patients in our study had a history of intrauterine death (IUD) and 5\% had history of still birth, implicating the need to screen women in pregnancy to prevent such disasters in the future pregnancies. There has been very limited data regarding these factors in association with chlamydial positivity in other studies. As there are no factors explaining the cause of IUD/ still birth in these patients, Chlamydia can be a significant risk factor. One study conducted in Assam, India by Mohan DG et al, ${ }^{17}$ has taken these factors under consideration but showed no positivity in the group.

Co-infection of Chlamydia trachomatis with other STI's was seen in $25 \%$ of the cases. $20 \%$ had co infection with Candida albicans and $5 \%$ had coinfection with $H I V$. In a study reported in North India by Malhotra $\mathrm{M}$ et al, ${ }^{8}$ showed co-infection with Candidiasis in $10.9 \%$ of the cases. Study by Joyee AG et $\mathrm{al}^{9}$ Chennai, showed HIV co-infection with C.trachomatis in $29.5 \%$ cases. However, our study has shown a low co-infection rate with HIV. This probably might be because $H I V$ was not routinely tested on all the patients in our study group. There is however, significant literature which prove the high rate of $H I V$ co-infection. Therefore, controlling and treating Chlamydia by screening women at an early age might be able to bring down the burden and transmission rate of $H I V$.

Co-infection with N.gonnorheae was not noticed in this study. Individuals infected with both C.trachomatis and N.gonnorheae shed larger number of C.trachomatis than those infected with Chlamydia alone. Co-infection between the two has been reported to range between 1.1 to $67 \% .^{21}$

Co-infection with other STIs highlights the importance of early laboratory diagnosis and specific treatment of the condition as they increase the risk many folds when infections exist together.

Primary prevention through life style modifications and health education can prevent both exposure and acquisition of genital chlamydial infections but has not gained popularity in the developing world. Secondary prevention through screening of sexually active women can detect chlamydial infections at an early stage and prevent the drastic sequelae of chlamydial infection. Also this study shows a high percentage of chlamydial infections which brings about the need to implement effective screening strategies, as this infection can be easily diagnosed with the latest noninvasive and rapid turnaround time tests like DFA. Tertiary prevention of chlamydial infection of the upper genital tract has largely failed because by the time the patient becomes symptomatic, substantial damage to the tubes would have occurred, leading to infertility.

\section{Limitations of the study}

There are certain limitations to our study. Ours is a hospital based study involving only the symptomatic group .Due to lack of accessthe test could not be compared with other specific tests like nucleic acid amplication tests or culture of the bacteria. But most of our laboratories lack these facilities except few elite laboratories situated in urban areas .Evaluation with larger sample size and for a longer duration is required to know the exact magnitude of the problem.

\section{References}

1. World Health Organization. Global incidence and prevalence of selected curable sexually transmitted infections-2008. Who [Internet]. 2012;1-28.[cited 2014 Aug 25] Available from:

2. http://www.who.int/reproductivehealth/publications/rtis/2 008_STI_estimates.pdf.

3. Malhotra M, Sood S, Mukherjee A, Muralidhar S, Bala M. Genital Chlamydia trachomatis: an update. Indian J Med Res. 2013;138:303-16.

4. Buckner LR, Amedee AM, Albritton HL, Kozlowski PA, Lacour N, McGowin CL et al. Chlamydia trachomatis 
Infection of Endocervical Epithelial Cells Enhances Early HIV Transmission Events. PLoS ONE 2016; 11:1-20. e0146663. doi:10.1371/ journal.pone.0146663.

5. da Silva Barros NK, Costa MC, Alves RR, Villa LL, Derchain SF, Zeferino LC et al . Association of HPV infection and Chlamydia trachomatis seropositivity in cases of cervical neoplasia in Midwest Brazil. J Med Virol. 2012;84:1143-50. doi: 10.1002/jmv.23312

6. Visnovsky J, Biskupska-Bodova K, Cabanova B, Kudela E, Dokus K. Early Fetal Loss and Chlamydia Trachomatis Infection. Gynecol Obstet. 2013;3:5-7.

7. Torrone E, Papp J, Weinstock H; Centers for Disease Control and Prevention (CDC). Prevalence of Chlamydia trachomatis genital infection among persons aged 14-39 years-Unites States, 2007-2012. MMWR Morb Mortal Wkly Rep. 2014;63:834-38.

8. Manavi K. A review on infection with Chlamydia trachomatis. Best Pract Res Obstet Gynaecol. 2006;20:941-51.

9. Malhotra M, Bala M, Muralidhar S, Khunger N, Puri P. Prevalence of Chlamydia trachomatis and its association with other sexually transmitted infections in a tertiary care center in North India. Indian J Sex Transm Dis.2008;29:82-5.

10. Joyee AG, Thyagarajan SP, Sowmya B, Venkatesan C, Ganapathy M. Need for specific and routine strategy for the diagnosis of genital chlamydial infection among patients with sexually transmitted diseases in India. Indian J Med Res. 2003;118:152-7.

11. 10.Ikeme AC, Ezegwui HU, Ikeako LC, Agbata I, Agbata E. Seroprevalence of Chlamydia trachomatis in Enugu, Nigeria. Niger J Clin Pract. 2011;14:176-80.

12. 11.Sood S, Mukherjee A, Bala M, Satpathy G, Mahajan $\mathrm{N}$, Sharma A, et al. A pilot study for diagnosis of genital Chlamydia trachomatis infections by polymerase chain reaction among symptomatic Indian women. Indian $\mathbf{J}$ Dermatol Venerol Leprol. 2012;78:443-7.

13. Sachdeva P, Patel AL, Sachdev D, Ali M, Mittal A, Saluja D. Comparison of an in-house PCR assay, direct fluorescence assay and the Roche

AMPLICOR Chlamydia trachomatis kit for detection of C. trachomatis. J Med Microbiol. 2009;58:867-73.

14. 13.Hermann B, Espinoza F, Villegas R, Smith G, Ramos A, Egger M. Genital chlamydial infection among women in Nicaragua: validity of direct fluorescent antibody testing, prevalence, risk factors and clinical manifestations. Genitourin Med. 1996;72:20-6.

15. Prathiba G, Joseph Pushpa Innocent D, Prabhu N. Prevalence of Chlamydia trachomatis infection in women in Chennai, India. Ann Biol Res. 2010;1:76-81.

16. Navarro C, Jolly A, Nair R, Chen Y. Risk factors for genital chlamydial infection.Can J Infect Dis 2002;13:195-207

17. Nessa K, Waris SA, Sultan Z, Monira S, Hossain M, Nahar $S$ et al. Epidemiology and Etiology of Sexually Transmitted Infection among Hotel-Based Sex Workers in Dhaka, Bangladesh. J Clin Microbiol. 2004;42:618-21.

18. Mohan DG, Borthakur AK. Seroprevalence of Chlamydia trachomatis in infertile women in a tertiary care hospital: A pilot study. Indian J Med Microbiol. 2015;33:331-2.

19. Dhawan B, Rawre J, Ghosh A, Malhotra N, Ahmed MM, Sreenivas V, et al. Diagnostic efficacy of a real time-PCR assay for Chlamydia trachomatis infection in infertile women in North India. Indian J Med Res. 2014;140:252-61.

20. Paavonen J, Eggert-Kruse W. Chlamydia trachomatis: impact on human reproduction. Hum Reprod Update. 1999;5:433-47.
21. Visnovsky J, Biskupska-Bodova K, Cabanova B, Kudela E, Dokus K . Early Fetal Loss and Chlamydia Trachomatis Infection. Gynecol Obstet. 2013;3:5-7.

22. Bala M, Mullick JB, Muralidhar S, Kumar J, Ramesh V. Gonorrhoea\& its co-infection with other ulcerative, nonulcerative sexually transmitted \& HIV infection in a Regional STD Centre. Indian J Med Res. 2011;133:3469. 